\title{
ON A NEW METHOD FOR THE GENERATION OF SOUND-WAVES. ${ }^{1}$
}

\author{
By Jul. Hartmann, \\ Dr. techn. Docent in the Royal Technical College, Copenhagen. \\ SYNOPSIS.
}

Pressure variation along a high-velocity air jet, produced by a pressure of 0.9 to $5 \mathrm{~atm}$. above normal, was determined with a simple Pitot tube, and the periodic intervals of instability were located (Figs. 2 and 5 ).

New Source of Sound; Resonator Actuated by a High-Velocity Air Jet.-It was found that the intervals of instability referred to above can be used for the generation of sound. (I) Bulb form. When the small orifice to a bulb is placed in one of these intervals so that the particles of air move in and out of the opening, pulsations like those of a siren are produced, the tones not being pure. The fundamental frequency depends on the volume of the bulb, the size of the orifice and the position in the jet, and may readily be varied from I/ 10 to 5,000 per second. (2) Cylindrical form. When a simple cylindrical resonator is placed with one end in an interval of instability, pure tones are produced except in certain positions when overtones may be present. The frequency is the fundamental of the resonator and may be made as high as 125,000 per second by using a very short tube, about $0.5 \mathrm{~mm}$. in length and in bore. With a hydrogen jet, the frequencies are, of course, 3.8 times as high. The efficiency of such sources is remarkable, intensities that are painful being readily produced. Practical forms of these generators are illustrated (Figs. 3 and 4 ).

\section{INTRODUCTION.}

IF air is allowed to stream from a container in which the absolute pressure is more than $1.9 \mathrm{~atm}$. - that is the overpressure is higher than $0.9 \mathrm{~atm}$. - through a hole or a short bore out into the free atmosphere, a jet is produced which exhibits a peculiar periodic structure. It was Mach and Salcher ${ }^{2}$ who first noticed this structure by photographing the jet. Mach explained it as due to stationary sound-waves and suggested an explanation as to how the waves were created. Later the jet was studied by R. Emden ${ }^{3}$-also by means of photography-and he produced a theory for the sound-waves. A direct investigation of the distribution of the pressure in the air-jet was carried out by Parenty ${ }^{4}$ by means of a sound which was introduced into the jet, and the same method, but with considerably improved apparatus, was employed by

${ }^{1}$ Extract from "Det kgl. Danske Videnskabernes Selskab. Mathm. fysiske Meddelelser," I., I3, I919.

2 Wied. Ann., 4I, p. I44, I89o.

3 Wied. Ann., 69, p. 264 and 426, r899.

4 Ann. Chem. Phys., I2, 289, I897. 
Stodola. ${ }^{1}$ Stodola's experiments have to a high degree contributed to elucidate the structure of the air-jet. Finally should be mentioned very important work done by Prand $\mathrm{tl}^{2}$ who succeeded in solving the differential equation for the wave-motion in the jet, and to whom we owe a series of exceedingly instructive photographs of the jet. Thus we now possess a fairly complete comprehension of the air-jet and especially it is now known that the periodical structure is closely connected with the fact of the velocity of the jet, with sufficiently high overpressure, being higher than that of the sound.

The Use of the Air-jet for the Generation of Sound-wayes.

The author has carried out a series of investigations on the jet by means of the arrangement shown in Fig. I. In this figure, the jet-forming

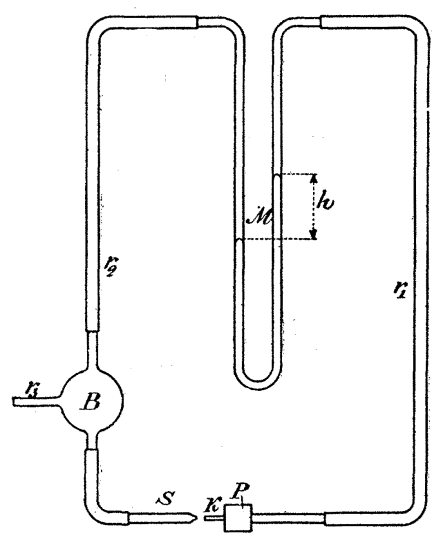

Fig. 1.
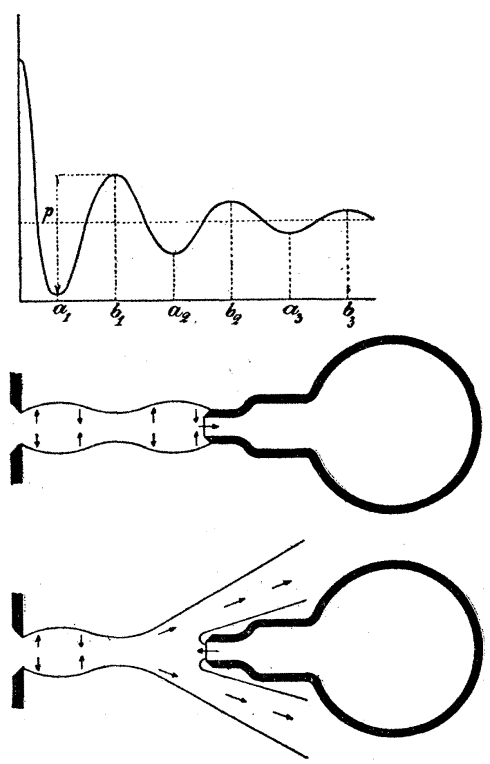

Fig. 2.

element is indicated by the letter $S$ and is shown as a drawn-out glass tube. $K$ designates the sound of a Pitot apparatus connected through the rubber tube $r_{1}$ with one of the branches of the U-shaped manometer $M$. The other branch of $M$ was, through $r_{2}$, connected with the bulb $B$, from which the air was flowing to $S$. In $B$ the air was kept at a constant pressure by means of a compressor, which through $r_{3}$ communicated with $B$. In the experiment an air-chamber of about $15 l$ was inserted between the bulb $B$, which had a volume of about $\frac{1}{2} l$, and the compressor.

1 A. Stodola, Die Dampfturbine, Berlin, I9 Io.

${ }^{2}$ Phys. Zs., 5, p. 599, 1904, and 8, 23, I907. 
In representing the pressure difference $h$, read on the manometer, graphically for varying positions of the sound $K$ out along the axis of the air-jet one gets a curve of the character shown in Fig. 2, $a$, provided the pressure in $B$ is lying between the above-mentioned critical and a certain higher value. The curve, as will be seen, gives a picture of the periodical structure of the jet. In comparing the curve with photographs of the jet it is found that the Pitot curve fairly truly represents the pressure-variations along the axis of the jet.

It was now found that the intervals $a_{1} b_{1}, a_{2} b_{2}, a_{3} b_{3}$ and so on, the intervals of instability as they are termed below, within which the pressure increases, may be used for the production of air-waves of two different types, which will be considered separately in the following. For the production of the one kind of waves a bulb, Fig. $2 b$, provided with a tube which is drawn out to a fine aperture, can be used. When this aperture is introduced into one of the intervals of instability, as indicated in the figure where the jet is drawn in correct position to the curve in Fig. 2, $a$, the bulb will periodically fill with, and again exhaust air. The motion of the air particles during the filling and the exhaust is schematically indicated in Fig. 2, $b$ and Fig. 2, $c$. The period of the phenomena depends on the size of the bulb, on the width of its mouth, on the position of this latter in the interval $a b$, and finally on the interval of instability, and on the pressure in that container from which the air flows to the jet. The larger the volume or the smaller the mouth, the longer the period. All periods from, say, Io sec. or more to $\mathrm{I} / 5,000$ sec. or less can easily be produced. The rapid oscillations of the air are of course heard as a tone. Same has the same unclear character as that of a siren. We shall in the following term the indicated air-oscillations as pulsations in order to distinguish them from the other kind of oscillations which may appropriately be called resonance-oscillations. The latter receive their designation from the fact that they are produced by introducing the mouth of a simple cylindrical resonator (oscillator) into one of the intervals of instability. The air will in this case oscillate mainly with the natural frequency of the resonator so that the produced regular sound-waves attain a wave-length of fully four times the depth of the resonator. Thus the frequency is now to a certain degree independent of the interval of instability as well as of the location of the aperture of the resonator in this latter, and of the pressure of the outflowing air, etc. The intensity of the oscillations however undoubtedly increases with the rise of pressure $p$ in the interval of instability. (See Fig. 2, a.) It is ordinarily very high due undoubtedly partly to the high density of the energy in the air-jet, partly to a comparatively very high 
efficiency of the generator. (Provisory measurements seem to prove that the efficiency at about 20,000 periods per sec. is as high as Io per cent.) As to the frequency, resonance-oscillations, i.e., pure acoustic oscillations of 100,000 periods per second or more, can easily be produced by means of a jet of atmospheric air. With a jet of hydrogen a frequency of more than 300,000 can be obtained. As a matter of fact the author has been able to produce intense sound-waves of 340,000 periods, and in all probability there will be nothing to prevent the production of still higher frequencies. The author therefore holds the opinion that the air-jet in connection with a resonator furnishes a means for producing intensive, pure acoustic oscillations of higher frequencies than has hitherto been possible.

\section{Practical Forms of the Acoustic Generator.}

As to the practical construction of an apparatus for the production of air-waves (pulsations of resonance-oscillations) according to the new principle, numerous possibilities present themselves. What is required is merely a means for inserting the aperture of the resonator or "pulsator" (Fig. 2, $b$ ) into the interval of instability and to center the aperture in relation to the jet. Fig. 3 shows a construction which is adaptable for

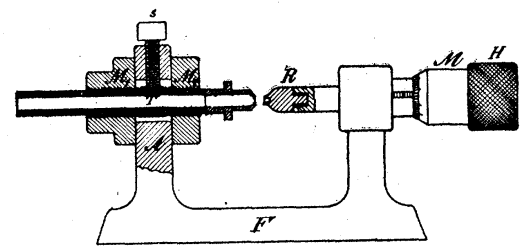

Fig. 3.

many purposes. The pressure-tube is here $T$, which has been extended with the jet-forming element $S . \quad T$ is carried through the wide bore in the vertical arm $A$ which is provided with plane sides. By means of two nuts $M_{1}$ and $M_{2}, T$ is fastened to $A$. By loosening one of these nuts the jet-hole may be centered relative to the mouth of the resonator $R$ by means of three screws $s_{1}, s_{2}, s_{3}$, of which only one, $s_{1}$, is shown in the figure. The resonator is made as a cylindrical bore, of approximately the same diameter as the jet-hole, at the end of a short piece of metal. The edges of the bore are sharpened as shown in the figure. The resonator, respectively the pulsator is screwed into the end of the micrometer screw $M$ and can by means of this be adjusted in one or the other of the above-mentioned intervals of instability of the jet. The screw and the 
Physical Review, Vol. XX, Second Series, December, 1922.
Plate I.

To face page 722 .

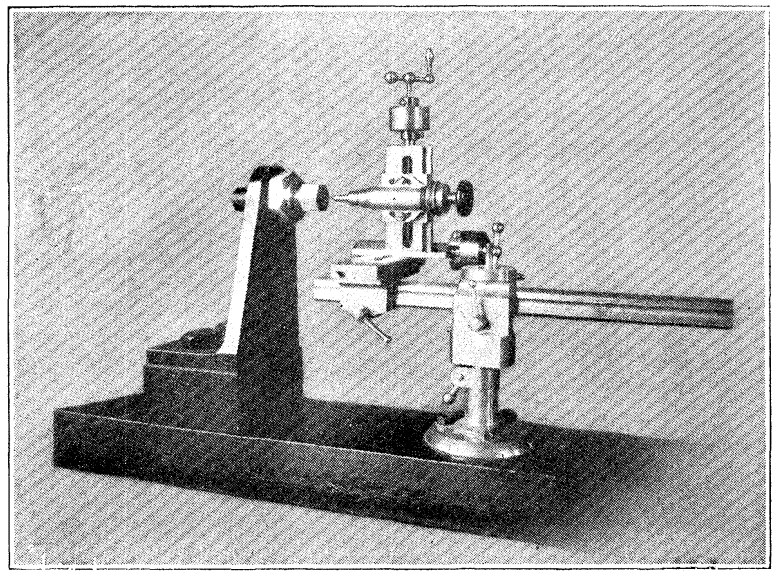

Fig. 4.

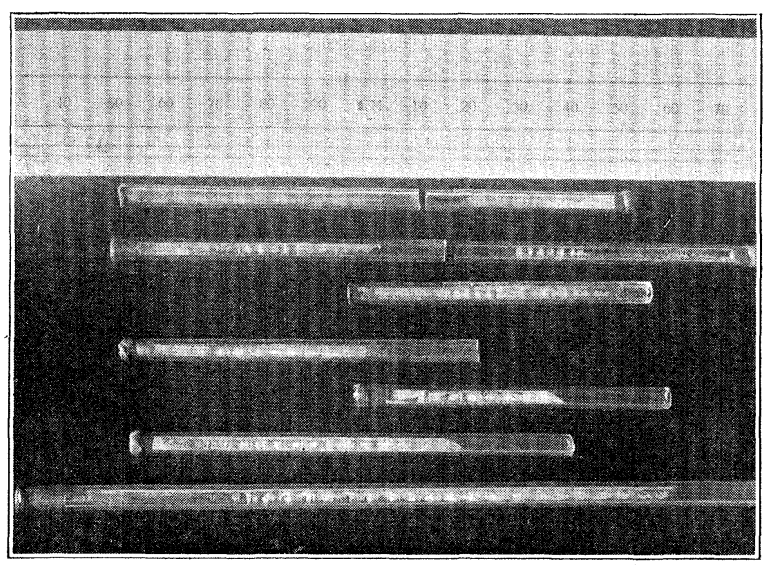

Fig. 7.

JULIUS HARTMANN. 
pressure-tube are connected through the baseplate $F$ which, if desired, may be attached to a heavier plate or to the apparatus in connection with which the acoustic generator is to be used.

During the fundamental experiments an apparatus was used which has in many respects proved practical. It is shown in Fig. 4. On a heavy iron beam is mounted a vertical stand through which is carried a short pipe. The pipe is at the one end provided with a head into which the jet-forming element is screwed. Another stand on the iron beam carries a horizontal guide rail. On this a combination of three slides may be moved towards or away from the jet-hole. The one slide carries a small chuck in which the resonator can be fastened. By means of two of the slides the resonator can be centered in relation to the jet. By means of the third one the aperture of the resonator can be carried into the interval of instability. Well suited for the jet-forming element is a glass tube drawn out to a short point. Equally suited is a conical bore in a metal plate and this latter form is of course easier to produce. Cylindrical bores are less satisfactory even if they have a conical inlet, as a certain pressure is lost in such bores.

In the event of no compressed-air plant being available, the air for the acoustic generator is taken from a compressed-air bottle. The bottle must of course be provided with a reduction valve. Between this and the generator is preferably inserted an air chamber of a capacity of some liters and provided with a valve by means of which the air supply to the generator may be stopped.

EXPERIMENTS ON THE Location of the Intervals of Instability.

In the following an extract will be given of the experiments, which were carried out as a basis for the new acoustic generator. Fig. 6 represents a curve showing the variation in the readings of the manometer in Fig. I out along the axis of an air-jet produced by an over-pressure of I4I.O cm. mercury and by a jet-hole of $0.535 \mathrm{~mm}$. The distances from the jet-hole, measured on a scale, were roo divisions are equal to $0.275 \mathrm{~mm}$., are shown as abscissæ, and as in Fig. 2, the ordinates represent the difference between the pressure in the container and in the jet. The great regularity in the periodical pressure-variations is especially to be remarked. The approximate location of the intervals of instability is shown in the figure. The second interval extends somewhat out over the press. --maximum, this is however only due to the uncertainty in the determination of the location. The extension of the intervals of instability was found by means of small "pulsators" made of glass tubes which were at the one end drawn out to a small aperture and at 
the other end closed by sealing-wax. In the examination of the first interval in Fig. 5, two pulsators of different sizes were used. Each of

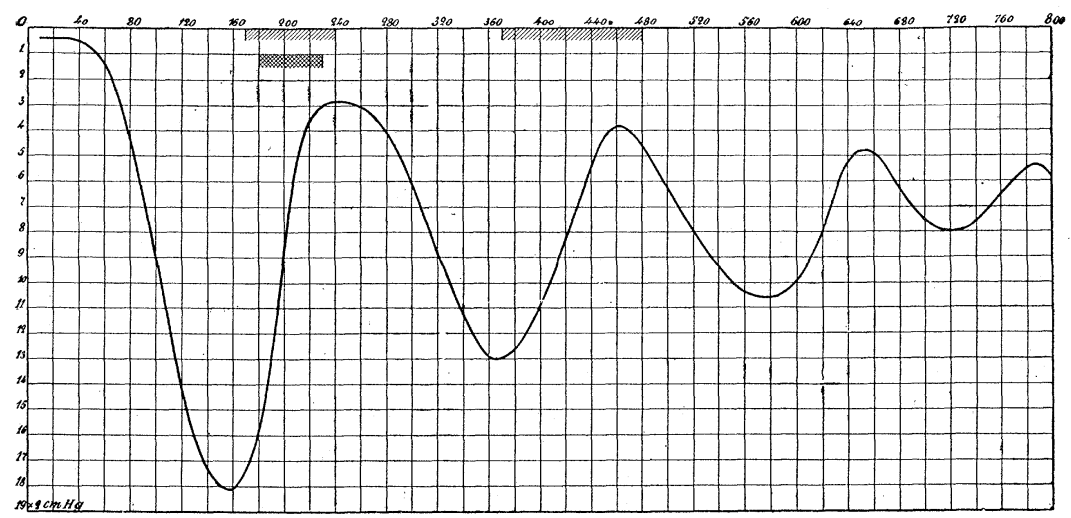

Fig. 5.

the two indicated intervals corresponds to its pulsators. The largest interval was found by means of the largest pulsator. The smallest pulsator had a volume of only some few $\mathrm{mm}^{3}$, the largest was not larger than that its pulsations could be heard as a tone. If the aperture of the

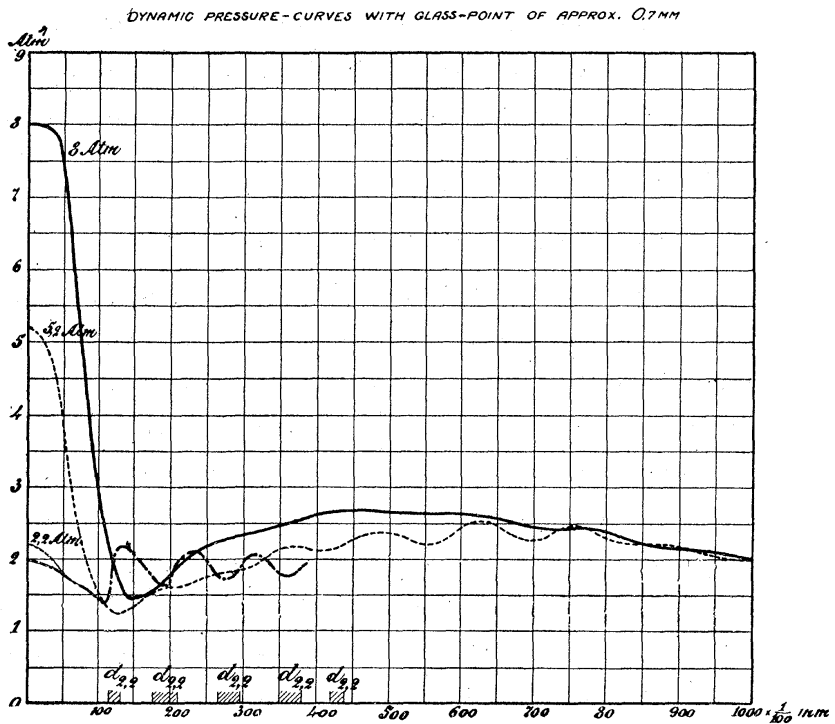

Fig. 6.

smallest pulsator was placed in the first part of the first interval (nearest the jet-hole) it would produce, within the small interval which is denoted in the figure by a double-arrow, a pure tone of about I00,000 oscillations per second. 
The periodical character of the pressure-variations in the jet is only observed for overpressures under a certain limit. Fig. 6 illustrates the changes when this limit is passed. At the lowest overpressure of 2.2 atm., the periodical pressure-variations and the sharply separated intervals of instability $d_{2 \cdot 2}, d_{2 \cdot 2}, \cdots$ again occur. Altogether five such intervals have been observed. Undue importance must not be attached to the limits of the intervals, as the determination of same was connected with great uncertainty. When the pressure is increased the intervals by and by are mingled up. At a pressure of 5.2 atm., an interval of

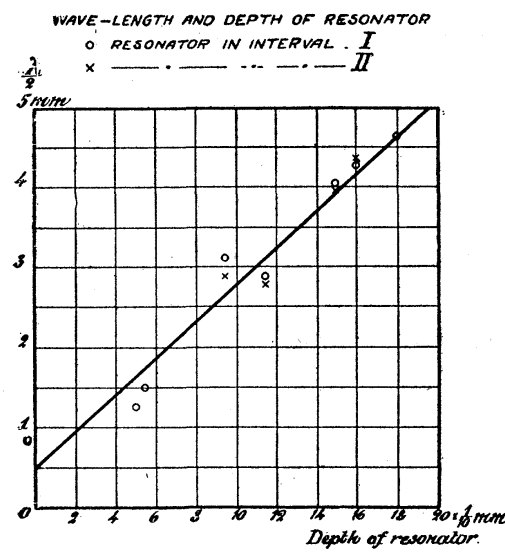

Fig. 8.

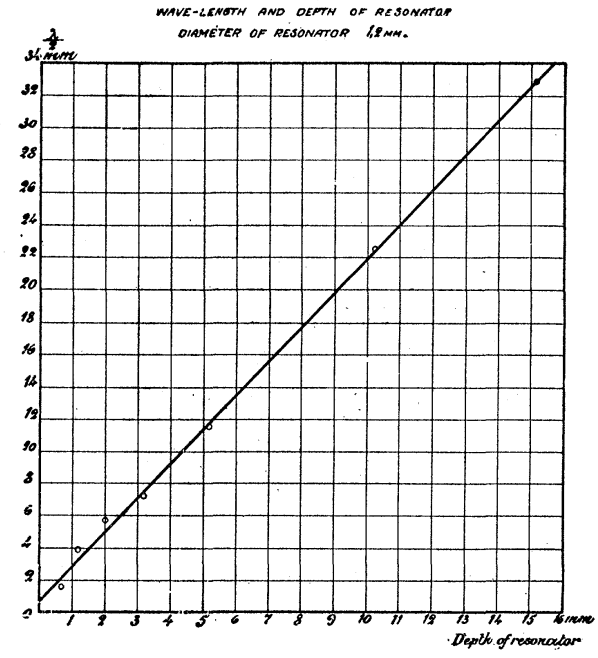

Fig. 9.

instability started at the abscissa 350 and extended to 620. Thereafter followed a smaller isolated interval from 660 to 700 . With a resonator (No. I3) which produced a rather high tone, the beginning of the interval of instability was observed as low as 230 and the interval extended to about 90o. Possibly there could be traced outside this interval a smaller one between 950 and 1050 .

\section{Experiments on the Dependency of the Number of Oscillations on the Dimensions of the Resonator.}

A number of resonators of the type shown in Fig. 3 were produced. The depth $d$ was measured and it was investigated how the produced oscillations depended on $d$. The wave-length $\lambda$ corresponding to the oscillations was measured by means of Kundt-tubes. Samples of the dust-figures are shown in Fig. 7 together with a millimeter seàle which makes it possible to determine the length of the waves. In Fig. 8 a curve is reproduced showing the variation of $\lambda / 2$ with $d$, for a number of 
very small resonators, all of which had an aperture of $0.7 \mathrm{~mm}$. The two series of observations correspond to the two first intervals of instability. The curve is, as will be seen, a straight line. In Fig. 9 are represented the results of a second experiment which was made with deeper resonators of $1.2 \mathrm{~mm}$. aperture. The curve is also here a straight line and may with the units of the figures be represented by the formula:

$$
\begin{aligned}
& \lambda=4.22 \cdot d+\mathrm{I} .2, \\
& \lambda=4.22(d+0.3) .
\end{aligned}
$$

The wave-length is thus, as may be expected, approximately 4 times the depth of the resonator. The divergence from 4 is perhaps due to the fact that the temperature of the oscillating air in the resonator is not the same as that in the Kundt-tube. The constant $\mathbf{I} .2$ in the first formula indicates the well-known fact that the oscillations in the resonator extend somewhat outside the mouth of the resonator. It is usually assumed that the extension outside the mouth is approximately o.6 time the radius of the resonator. In the second formula 0.3 should represent this extension in fairly good accordance with that assumed, 0.6 time the radius of the resonator 0.6 being 0.36 .

The frequency of the oscillations of the resonator is as already mentioned, mainly determined by the dimensions of the resonator, and especially by its depth. Hereby it is of course assumed that the resonator is always blown with the same kind of air. If a jet of hydrogen is used, the frequency is found to be, as is to be expected and as has already been indicated, about four times as high as with a jet of atmospheric air. For instance the following results were found:

\begin{tabular}{r|c|c|c|c}
\hline \multicolumn{1}{c|}{ Resonator. } & $\lambda / 2$ Air. & $\lambda / 2$ Hydrogen. & Proportion. & Remarks. \\
\hline $6 \ldots \ldots \ldots \ldots$ & $0.464 \mathrm{~cm}$. & $0.130 \mathrm{~cm}$. & 3.6 & \\
$8 \ldots \ldots \ldots \ldots$ & $0.145 \mathrm{~cm}$. & $0.050 \mathrm{~cm}$. & 3.0 & uncertain \\
$14 \ldots \ldots \ldots$ & $3.47 \mathrm{~cm}$. & $0.925 \mathrm{~cm}$. & 3.5 & \\
\hline
\end{tabular}

The proportion between the wave-lengths (measured in the same medium) should be 3.8 . If it is perhaps somewhat lower, it may be because the technical hydrogen used was not quite pure.

The intensity of the here-considered resonance-oscillations is, as already stated, very large, notwithstanding the smallness of the resonator. The possibility of making the resonator very small, especially of a small diameter, explains how it comes that the frequency can be made as high as is actually the case. A small diameter means a small value of the constant term in the formula for the wave-length. Now it is obvious 
that the smaller this term is the smaller values or the higher frequencies can be obtained by diminishing the depth $d$ of the resonator. By way of illustration we may consider a resonator of a depth $0.5 \mathrm{~mm}$. With such a resonator the diameter can at least be made as small as $0.5 \mathrm{~mm}$. The constant term will in this case be

$$
\frac{0.6 \cdot 0.5}{2}=0,15 \mathrm{~mm}
$$

Thus the effective depth of the resonator is $0.65 \mathrm{~mm}$. which gives

$$
\frac{320,000}{4.065}=123,000 \text { periods per sec., }
$$

the velocity of sound being $320,000 \mathrm{~mm}$. $/ \mathrm{sec}$. With a hydrogen-jet the frequency would be $3.8 \cdot 123,000=476$, o0o periods per sec., thus very nearly half a million per sec. It may be remarked that oscillations of very high frequencies can easily be produced as first overtones of oscillators of greater depth. Some observations are stated in the table below. The first overtone is produced by adjusting the aperture of the resonator right up to that limit of the first interval of instability which is nearest to the jet-hole. In the indicated experiments the highest frequency is, as will be seen, about I20,000 periods per sec.

\begin{tabular}{r|c|c|c}
\hline \hline Resonator. & $\lambda / 2$ Fundamental Note. & $\lambda / 2$ Overtone. & Proportion. \\
\hline $22 \ldots \ldots \ldots \ldots \ldots \ldots$ & $1.20 \mathrm{~mm}$. & $0.400 \mathrm{~mm}$. & 3.0 \\
$7 \ldots \ldots \ldots \ldots \ldots \ldots$ & $0.65 \mathrm{~mm}$. & $0.205 \mathrm{~mm}$. & 3.2 \\
$12 \ldots \ldots \ldots \ldots \ldots$ & $0.49 \mathrm{~mm}$. & $0.142 \mathrm{~mm}$. & 3.4 \\
\hline
\end{tabular}

The intensity of the resonance-oscillations may be illustrated by the following example. A resonator of about 24,000 periods per sec. and with an aperture of $\mathrm{I} .7 \mathrm{~mm}$., was adjusted in an air-jet produced with a bore of $\mathrm{I} .5 \mathrm{~mm}$. With an overpressure of $4-5 \mathrm{~atm}$. very good dust-figures were obtained at a distance of about $\frac{1}{2} \mathrm{~m}$. from the resonator when a Kundt tube of $4 \mathrm{~mm}$. aperture was used. With a resonator of about Io,OOO periods per sec. and with an aperture of $1.5 \mathrm{~mm}$. the produced tone was even with rather small overpressures almost unbearably intensive and left the observer with a physiological after-effect of several hours' duration.

The Royal Technical College, Copenhagen. 


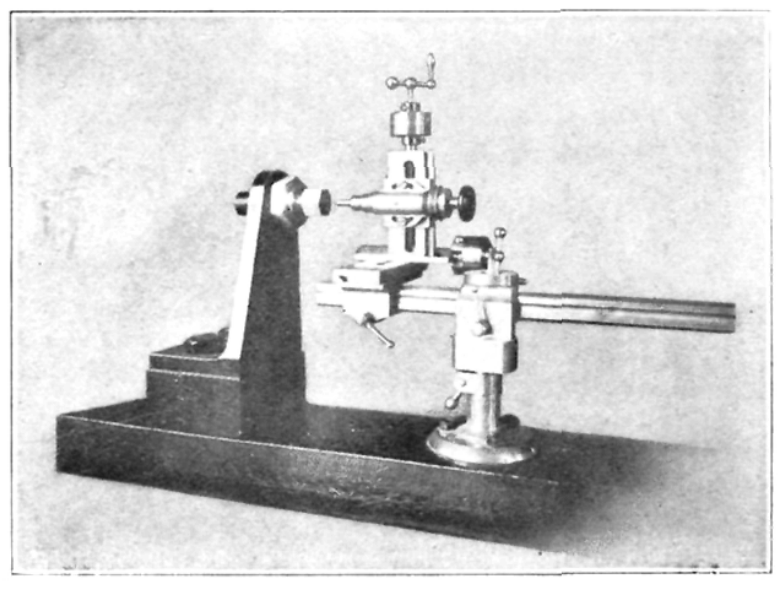

Fig. 4. 


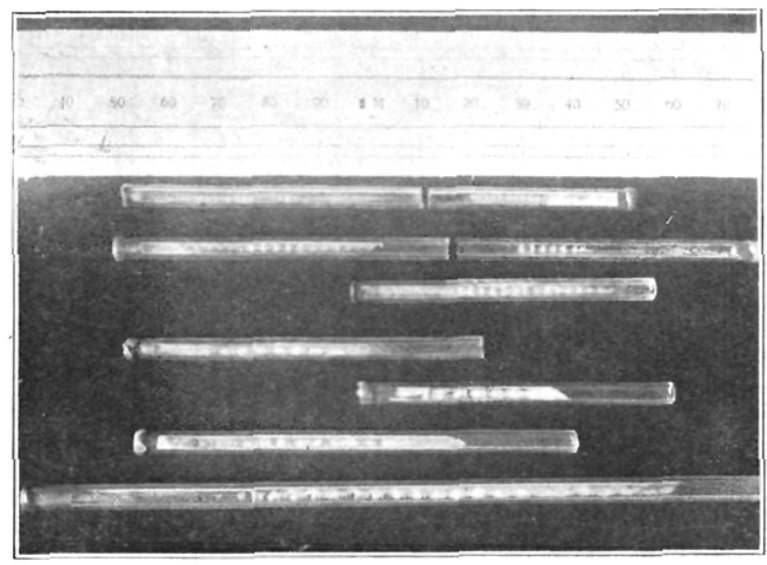

Fig. 7. 